\title{
TWO INFANT VISION SCREENING PROGRAMMES: PREDICTION AND PREVENTION OF STRABISMUS AND AMBLYOPIA FROM PHOTO- AND VIDEOREFRACTIVE SCREENING
}

\author{
JANETTE ATKINSON ${ }^{1}$, OLIVER BRADDICK ${ }^{1}$, BILL BOBIER $^{1}$, SHIRLEY ANKER $^{1}$, \\ DAVID EHRLICH ${ }^{1}$, JOHN KING $^{1}$, PETER WATSON $^{2}$ and ANTHONY MOORE ${ }^{2}$
}

\begin{abstract}
SUMMARY
Two infant vision screening programmes on total populations in the Cambridge Health District have been designed to identify manifest strabismus and strabismogenic and amblyogenic refractive errors at 7-9 months of age. The first, completed, programme used the isotropic photorefractor with cycloplegia together with a standard orthoptic examination. The second, current, programme uses the VRP-1 isotropic videorefractor to identify infants with accommodative lags which are followed up by refraction under cycloplegia. Both programmes show good agreement between infants identified at screening and retinoscopic refractions at follow-up, showing that photo- and videorefraction (with or without cycloplegia) can be effective methods for screening for ametropia in infants and young children. In each programme 5-6\% of infants showed abnormal levels of hyperopia $(\geqslant 3.5 \mathrm{D}$ in any meridian), less than $1 \%$ showed anisometropia $\geqslant 1.5 \mathrm{D}$; very few infants $(0.25 \%)$ showed $-3 \mathrm{D}$ myopia or greater. Less than $1 \%$ showed manifest strabismus. Hyperopic and anisometropic children entered a randomised controlled trial of partial refractive correction. All children identified at screening, alongside appropriate control groups, are extensively followed up to age 4 years. The first programme has found that children who were hyperopic in infancy were 13 times more likely to become strabismic, and 6 times more likely to show measurable acuity deficits by 4 years, compared with controls. Wearing a partial spectacle correction reduced these risk ratios to $4: 1$ and $2.5: 1$ respectively. The impaired acuity can be attributed, in part, to meridional amblyopia resulting from persisting astigmatism. Both hyperopic and myopic infants
\end{abstract}

From: ${ }^{1}$ Visual Development Unit, London and Cambridge, and University College London; ${ }^{2}$ Department of Ophthalmology, Addenbrooke's Hospital, Cambridge UK.

Correspondence to: Prof. J. Atkinson, Department of Psychology, University College London, Gower Street, London WC1E 6BT, UK. showed refractive changes in the direction of emmetropia between 9 months and 4 years. Wearing a partial spectacle correction did not affect this process of emmetropisation, but does provide the possibility of reducing the incidence of common pre-school vision problems.

Strabismus and amblyopia, both as a consequence of refractive error and/or strabismus, are not necessarily the most individually serious of visual problems in childhood, but they are important because they are the most prevalent in developed countries. Estimates of incidence from different studies and different populations have been quite variable, but in the United Kingdom 3\% of preschool children would be a conservative estimate.

Normally, accurate convergence and binocular fixation are maintained by the detection of binocular disparity. In manifest strabismus this mechanism fails. In principle, this could be for either (or both) of two broad reasons. The strabismic child might lack the neural mechanisms necessary to detect binocular disparity. Alternatively, disparity processing might be intact but its control over vergence overwhelmed by some other factor. If lack of binocular disparity detection is not the cause of strabismus but only secondary to it, what are the forces that can override the control of vergence by disparity? Clinically, a large proportion of children with convergent strabismus are considered to be accommodative esotropes with associated hyperopia. According to this theory, the high accommodative demand in hyperopia means that accommodative vergence is a stronger force than normal, in some cases so strong that it breaks down the maintenance of convergence on the target by disparity control and the child squints.

This theory leaves several questions unanswered. First, why do some hyperopic children become

Eye (1996) 10, 189-198 C 1996 Royal College of Ophthalmologists 
strabismic while others do not? In adults, it is possible to measure the strength of accommodative convergence, expressed as the 'AC/A ratio', which shows large variations from one individual to another. Presumably a large AC/A ratio in a hyperope disposes the child towards accommodative esotropia, but the developmental foundations of this ratio are unknown and at present there are no means of measuring it in infants at the stage when the strabismus may be originating. This leads to a second question: Why should the hyperopic child's accommodative vergence not adjust to the amount required for the individual's refractive error? Visuomotor systems are generally highly flexible in adjusting to the relationship between sensory input and required output; why should this system be insensitive to the individual's particular correspondence between the vergence required to null a target's disparity and the accommodation required to minimise its blur? This is especially surprising, given the plasticity required to cope with changing vergence requirements as the child's head grows and interocular separation increases. Furthermore, the accommodation-vergence relationship has been shown experimentally to be plastic in adulthood. ${ }^{1}$ However, an interesting feature of this plasticity is that it has been demonstrated by increasing the required vergence (as would be required to cope with a growing head) but not yet in the opposite direction (as would be required to cope with hyperopia).

The idea of accommodative esotropia derives from the fact that the child who presents to an ophthalmologist with convergent strabismus is often hyperopic, and that spectacle correction of this error may reduce the angle of strabismus. However, in clinical practice the child's refractive history before the onset of the strabismus is usually unknown. It is possible that some strabismics, who are initially hyperopic, have lost this refractive error by the time they clinically present. This possibility is supported both by Ingram et al.'s study ${ }^{2}$ and that described below.

The large-scale Cambridge studies described here were designed to test this possibility further, using a relatively new refractive screening method to detect hyperopia and other refractive errors in infancy. If the theory of accommodative esotropia is correct, then it should be possible not only to detect the hyperopia but also, by prescribing spectacles that reduce the required accommodative effort, to help prevent the later onset of strabismus. Our studies therefore include a trial of this intervention strategy.

Previous large-scale studies of refractive errors in infants, and their predictive value for strabismus and amblyopia, have been carried out by Ingram..$^{2-4}$ These used retinoscopy as the screening technique, which makes heavy demands on the time of very skilled personnel and requires a certain degree of co- operation on the part of the child. Retinoscopy is consequently costly and not optimal for widespread screening. In the present Cambridge studies we used isotropic photorefraction and videorefraction, carried out by trained orthoptists, a method which has been previously validated against retinoscopy. Further independent validations of isotropic videorefraction against retinoscopy have been carried out by Spanish and Russian clinical teams. ${ }^{6,7}$ Compared with retinoscopy, photo- and videorefraction requires less highly trained personnel and less cooperation on the child's part; it is therefore a relatively robust and inexpensive method.

In the isotropic method ${ }^{5,8-11}$ the centrally placed light source generates a 'blur ellipse' whose orientation reflects the axis of any astigmatism present. It thus measures the refractive state in all meridians equally well - an advantage in testing young infants among whom significant astigmatism is common. The alternative method of 'eccentric photorefraction' or 'photoretinoscopy'12-14 measures the meridians along which the light source(s) are placed, and interpretation of astigmatic errors in other meridians is complex. ${ }^{15}$

The first of the Cambridge screening programmes, $5,10,16,17$ now completed, screened for refractive error by photorefraction under cycloplegia. However, the convenience and acceptability of a screening procedure would be greater if cycloplegia were not required, and infants' accommodative behaviour may be as significant as their cycloplegic refractions. In the second, current, programme, therefore, we have performed the initial screening with freely accommodating infants. The second programme ${ }^{18-20}$ has also taken advantage of the availability of the VPR-1 videorefractor. This instrument uses the same optical configuration as isotropic photorefraction but captures the image in a digital framestore from which the operator can immediately make measurements on a computer screen. This has the advantage over photorefraction that test results can be reported to the family and used for a referral decision at the time of screening.

\section{POPULATIONS, METHODS AND PROTOCOLS IN THE CAMBRIDGE SCREENING PROGRAMMES}

\section{Screening Procedure}

First Programme. Every infant living in the City of Cambridge over a 2-year period was sent an appointment to attend at a local Well Baby Clinic, when the infant was between 6-8 months post-term age. A total of 3166 infants $(74 \%$ of those sent appointments) were screened. A trained orthoptist conducted the screening tests, which consisted of a basic orthoptic examination (Hirschberg test, cover test, ability to overcome $20 \Delta$ prisms) and, following 
cycloplegia with $1 \%$ cyclopentolate, isotropic photorefraction. This involved taking three photographs on $35 \mathrm{~mm}$ colour transparency film with different settings of the camera focus. Infants were referred for follow-up if the photorefraction revealed marked hyperopia, myopia and/or anisometropia, or if strabismus or any ocular pathology was evident. Fuller details of the procedures for screening and follow-up, and of the validation of the photorefraction measure, have been published. ${ }^{5}$

The criteria for follow-up were as follows:

1. Any infant showing hyperopia over $+3.5 \mathrm{D}$, in one or more meridians.

2. Any infant showing myopia of $-2 \mathrm{D}$ or greater in any meridian.

3. Any infant showing anisometropia, i.e. a difference of $1.5 \mathrm{D}$ or more between corresponding axes.

4. Any infant showing any orthoptic or ocular problem, e.g. strabismus, ptosis.

Second Programme. The population in the second programme was similar to the first, being all the children in the Cambridge District born in a 2 year period, with screening at around 8 months of age. A total of 5923 children were sent appointments and 5091 attended $(86 \%)$. The incidence in this total population of a first-degree relative who had been treated for strabismus and/or amblyopia was $10 \%$.

The screening consisted of a full orthoptic examination by a senior orthoptist and four sets of videorefractive images (i.e three images taken in each set) using the VPR-1 without cycloplegia. Two sets were made with the camera and fixation target at $75 \mathrm{~cm}$ (the most sensitive distance for detecting hyperopic focus) and two sets at $100 \mathrm{~cm}$ (the most sensitive distance for detecting myopic focus). Since in previous studies we had found a significant relationship between non-cycloplegic accommodation (accommodative lag) and cycloplegic refraction, ${ }^{21,22}$ the goal has been to use non-cycloplegic measures at screening to predict significant refractive errors at follow-up.

The criteria for follow-up in the second programme were as follows:

1. Any infant showing an accommodative lag such that any meridian had a hyperopic focus greater than $+1.5 \mathrm{D}$, on either of the tests at $75 \mathrm{~cm}$ distance.

2. Any infant showing myopic accommodation of $-3 \mathrm{D}$ or greater in any meridian, on both tests at $100 \mathrm{~cm}$ distance.

3. Any infant showing anisometropia of $1.5 \mathrm{D}$ or greater between corresponding meridians, on any set of images.

4. Any infant showing any orthoptic or ocular problem.
In addition, in both programmes, a control group, who met none of the criteria for follow-up, were selected randomly from the same clinic as children meeting the refractive criteria for follow-up, and were followed alongside the potentially abnormal groups.

\section{Follow-up from Screening for Both Programmes}

The first follow-up occurred at around 9 months and included a fuller orthoptic examination, photorefraction or videorefraction prior to cycloplegia to examine accommodative performance, cycloplegic refraction by both photo/videorefraction and retinoscopy, and examination of the fundi. The first programme also included acuity testing by forcedchoice preferential looking (FPL) in this visit. For children in whom a refractive error was confirmed, there were repeated follow-ups at 4-6 month intervals, primarily concerned with monitoring refractive changes, acuity and any changes in orthoptic status. The second programme also includes developmental testing of other aspects of functional vision, language and motor behaviour to examine the general developmental correlates of refractive error. However, the follow-up of the second programme is currently still in progress and so these aspects of the study are not reported here.

In the first programme, at 4 years there were careful tests of the child's acuity and binocular vision. These tests provide the main outcome measures of this study. (Similar tests are intended for the second programme also.) Children who at any stage in the screening or follow-up showed manifest strabismus, extreme refractive error ( $+6 \mathrm{D}$ or more hyperopia, 2 $\mathrm{D}$ or more anisometropia, $-3 \mathrm{D}$ or more myopia), demonstrable amblyopia or ocular pathology were referred directly to the regular hospital eye clinic for treatment and follow-up information was derived from their examinations there.

Four main questions in these studies will be considered here:

1. What is the incidence of strabismus and significant refractive errors in normal populations of infants around 8 months of age? Here we will compare data from the two programmes.

2. How does refraction change with age? Here we will consider emmetropisation in infants initially identified with significant hyperopia or myopia in the first programme.

3. Are there identifiable strabismogenic and amblyogenic factors? Here we will consider whether hyperopic refractive errors are a precursor of later strabismus and/or poor vision in the first programme. Data on this question are not yet complete from the second programme.

4. Can intervention in the form of a spectacle correction for infant refractive errors reduce the 
risk of strabismus and amblyopia? Here we report results completed in the first programme from a randomised control trial of spectacle correction.

The second programme is intended to provide further information on questions 2-4 but this must await the group, screened in 1993-5, reaching the age for the outcome measures.

\section{Spectacle Corrections}

Spectacle correction was tested as a preventive intervention by randomly assigning infants in the hyperopic group to 'spectacles' and 'non-spectacles' groups. The spectacles were prescribed, on the basis of cycloplegic retinoscopic refraction, according to a defined protocol for partial correction:

Sphere: $1 \mathrm{D}$ less than least hyperopic meridian (corrections under 1.5 D were not prescribed)

\section{Cylinder.}

Up to 2 years of age: half of astigmatic error if over

\section{$2-3.5$ years:} $2.5 \mathrm{D}$

Over 3.5 years: half of any astigmatic refractive error

Prescriptions were checked frequently, and the Programme 1

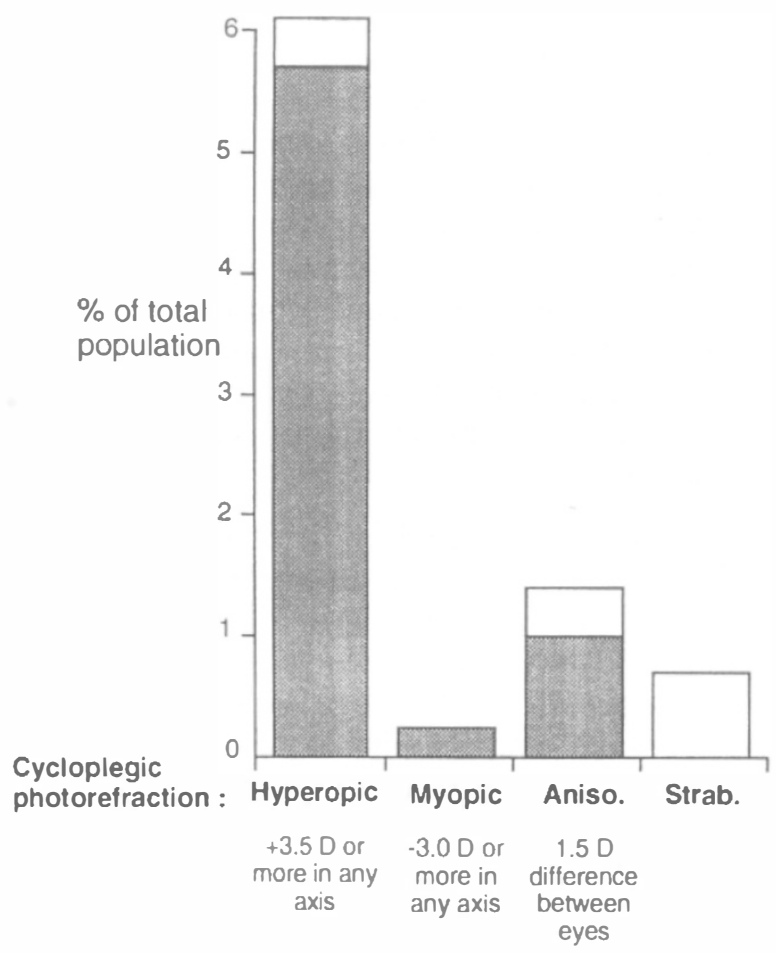

protocol above minimised the risk that a child whose refraction changed between follow-ups might be temporarily over-corrected. On each follow-up, the child's wearing of the spectacles was monitored by close but sympathetic questioning of the parents. This enabled us to measure compliance. We took as our working definition of 'wearing spectacles' any infant who was reported to wear the spectacle correction for $50 \%$ or more of their waking hours throughout the period in infancy when they were prescribed spectacles. The compliance level in the first programme was around $70 \%$ of those prescribed corrections.

\section{RESULTS}

Early Detection of Refractive Errors and Strabismus Fig. 1 illustrates the distribution of refractive errors (first programme) and accommodative lag (second programme) of those identified at screening. As can be seen from this figure, the great majority of the ametropias identified in screening were confirmed on the fuller examination at follow-up using cycloplegic retinoscopy (shaded areas in Fig. 1).

The largest single category of refractive errors was the infants showing hypermetropia (5-6\%). As would be expected, the non-cycloplegic screening in

\section{Programme 2}

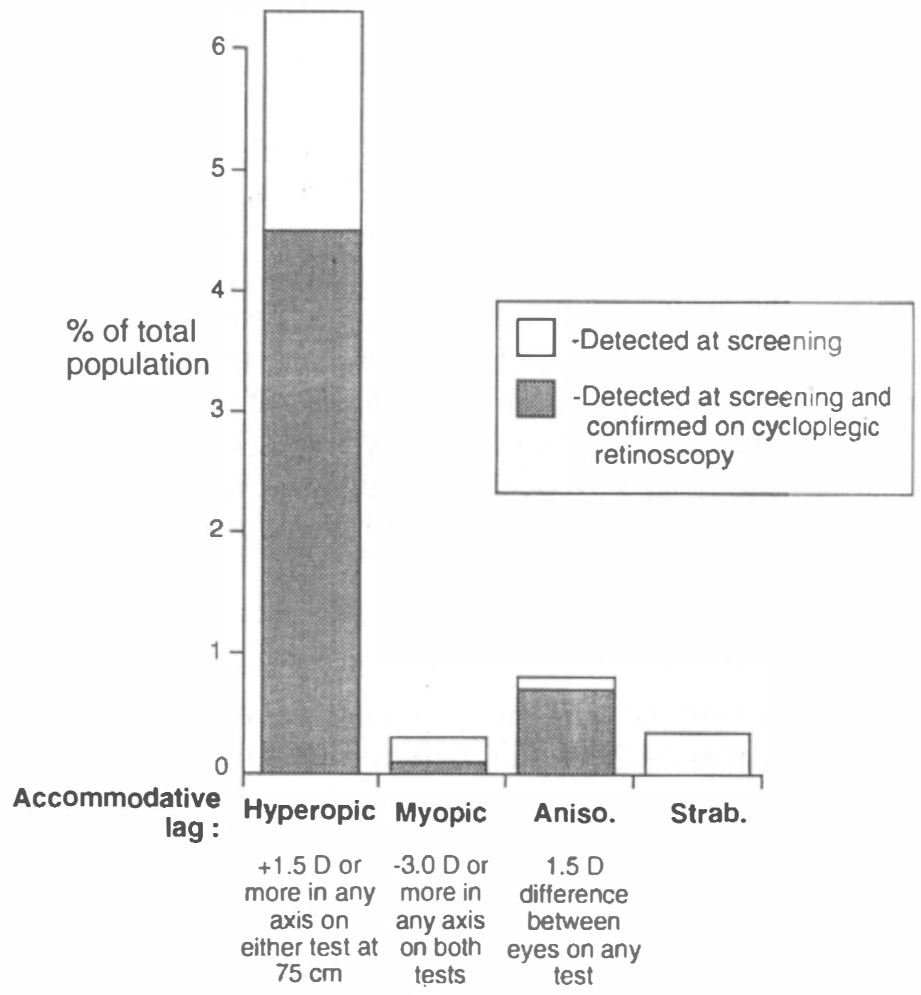

Fig. 1. Histograms showing the incidence in the population of significant refractive errors and strabismus, detected at screening (6-8 months of age) in the two programmes. 'Confirmation' for the second programme refers to the group who, detected on non-cycloplegic criteria, were found to meet the same cycloplegic criteria as those stated for the first programme. (The +3.5 D hyperopes in the first programme, excluded in some previous published analyses, are included here for compatibility between the two data sets.) 
the second programme identifies these less completely than does cycloplegic testing, but over $70 \%$ of those meeting the screening criterion of accommodative lag prove to meet the cycloplegic criterion of $+3.5 \mathrm{D}$ or greater. It should be noted that we have not included a separate category for astigmatism. Substantial degrees of astigmatism are common at the age we screened, with over $50 \%$ of infants having more than $1 \mathrm{D}$ of astigmatism ${ }^{9,10,23,24}$ and in most cases this largely disappears over the first 2 years. ${ }^{25}$ However, since the criterion for our hyperopic group was a refraction of $+3.5 \mathrm{D}$ or over in any meridian, and since even our normal group is on average over $+1 \mathrm{D}$ hyperopic, infants with large astigmatisms are likely to appear in the hyperopic group.

We should also note the small size of the group (around $0.5-1 \%$ ) found to be already strabismic at this age. In the first programme we were concerned that our screening might be omitting a strabismic group who had already seen an ophthalmologist before 6 months and who therefore did not attend screening. We therefore investigated a sample of 100 infants out of the approximately $25 \%$ who had not attended their appointments; only 2 were found to have been referred to a hospital eye clinic with possible strabismus, and in neither case was this confirmed. Any bias arising from differential attendance must therefore be very small, and the true figure for manifest strabismus before 6 months in this population is almost certainly below $1 \%$. It is of course possible that this group of very early onset strabismics differ fundamentally from those of later onset in their aetiology and precipitating mechanisms. Some have argued that a fundamental lack of the ability to develop disparity detectors may characterise the early onset group, but there is little evidence to support this view (see Braddick ${ }^{26}$ ).

\section{Changes in Refraction with Age}

How far do refractive errors identified at 6-8 months persist? Fig. 2 shows a summary analysis of data on refractive change from the first programme. The control children are showing, overall, very little change in their refraction between 8.5 months and 3 years, with the main change being reduction in the small degree of astigmatism present in many infants in the first year of life. Insofar as changes in this age range are generally in the direction of emmetropia, the control group have relatively little scope for change, since their average refraction at 8.5 months is already close to emmetropia (a small degree of hypermetropia in mean spherical equivalent).

The hyperopic groups, however, present a quite different picture. Overall there a strong trend for their hyperopia to decline. However, even at 3 years the hyperopic groups are still significantly more hyperopic than the controls $(p>0.05)$. There is no

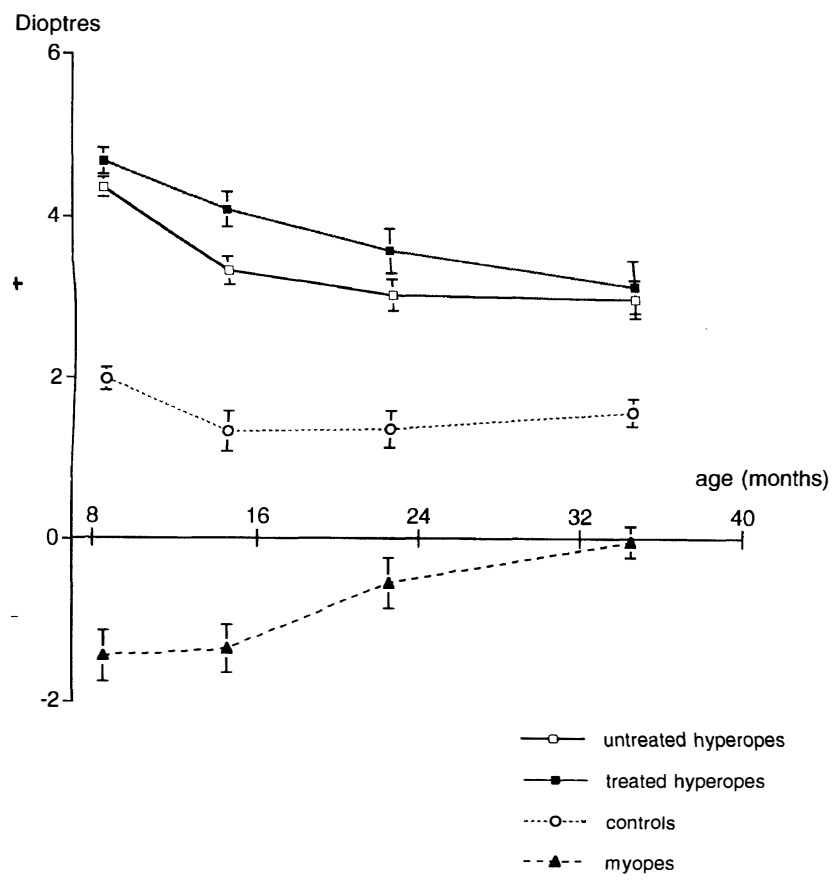

Fig. 2. Changes in refraction with age for each of four groups in the first screening programme: (1) control infants; (2) hyperopic infants not wearing spectacles; (3) hyperopic infants wearing spectacles; (4) 'myopic' infants (i.e. any axis $\leqslant-0.5 \mathrm{D}$ on initial cycloplegic refraction - a lesser criterion than the referral criterion defined for the main study). The highest hyperopic axis of the right eye is plotted for the hyperopes and controls; the highest myopic axis of the right eye is plotted for the myopic group. Error bars show SE of the mean.

evident difference in the rate of decline between those hyperopes who wore a partial correction and those who were uncorrected. There has been considerable discussion of the role of active visual feedback in controlling the development of the eye, which may act to optimise image quality and hence drive a process of emmetropisation (see discussion by Banks ${ }^{27,28}$ and Hung et al. ${ }^{29}$ ). The correction of image defocus by spectacles might reduce the error signal in such a feedback loop, and therefore reduce the developmental trend towards emmetropia. The present data, however, show no consistent effect on emmetropisation of wearing a spectacle correction.

It should be remembered that the protocol for spectacle prescription gave only a partial correction of hyperopia, and in particular there was cylinder corrections of only the largest astigmatism. Thus, even in the corrected hyperopes there would be some scope for accommodative adjustments and for activation of a feedback process. The practical conclusion is that concern about emmetropisation need not be an obstacle to the partial correction of hyperopic refractive errors in young children.

Fig. 2 also shows the reduction in myopia in the infants who were detected with small degrees of myopia; that is, the process of emmetropisation acts at both ends of the scale. A fuller account of these 
data has been published elsewhere. ${ }^{30}$ The myopic group also showed a reduction in astigmatism with age.

\section{Predictive Value of Refractive Screening for Later Strabismus and Amblyopia; Controlled Trial of Treatment}

We should not assume that an early refractive error necessarily of itself represents a visual deficit. Rather, we must ask whether identification of early refractive errors can predict lasting, functionally significant vision problems. We have been concerned with two aspects of the outcome at 4 years: binocularity and acuity. The main measure of binocularity is presence or absence of strabismus, although all children were also given the TNO random-dot stereo test. The two acuity tests were both conducted with the child wearing any appropriate refractive correction (in particular correcting any remaining astigmatism) even if the child was not wearing spectacles regularly as part of the trial, so failures are not simply a consequence of optical blur at the time of testing. Each eye was tested separately. The 'single-letter acuity' was obtained using the Sheridan-Gardiner letter matching test ${ }^{31}$ modified by us for a testing distance of $3 \mathrm{~m}$ rather than the standard $6 \mathrm{~m}$. 'Failure' was defined as $6 / 9$ or poorer with either eye. However, it is well known that testing with single optotypes does not satisfactorily reveal problems of amblyopia, where 'crowding' effects can be very marked (e.g. Hilton and Stanley ${ }^{32}$ ). Since a conventional Snellen chart is not practical at this age, we have devised a letter- matching task which is analogous to the SheridanGardiner test but presents the letters in a 'crowded' context (the Cambridge Crowding Cards). ${ }^{33-35}$ For adults, acuity estimates using the Cambridge Crowding Cards agree well with Snellen measures of acuity. On the basis of testing visually normal children, we set $6 / 12$ or poorer as the criterion of failure on this multiple-letter test. Fig. 3 shows the results from these outcome measures for controls, hyperopes who complied with spectacle wear and those who were either not given spectacles or did not wear them consistently.

As can be seen from Fig. 3, 21\% (16/76) of the children who had been hyperopic initially and had not worn a correction throughout infancy became strabismic, compared with $1.6 \%(2 / 123)$ of the control group (emmetropic in infancy) and 6.3\% (3/48) of the treated compliant group. The difference in outcome between treated and untreated groups is statistically significant (chi-squared test, $p<0.05$ ), but the difference between the control group and the treated hyperopes did not reach significance (although the incidence is higher in the latter group).

The pass/failure rates for the two acuity measures tests are shown for the three groups in Figs. 4 and 5. On the single-letter matching test $37.5 \%$ (27/72) of the untreated hyperopic group failed in one or both eyes, compared with $9.5 \%(4 / 42)$ of the treated hyperopes and $5.6 \%(7 / 123)$ of the control group. The untreated group were significantly worse than the other two groups (chi-squared, $p<0.001$ in both cases). On the crowding test $68 \%(47 / 69)$ of the untreated group, $28.6 \%(12 / 42)$ of the treated

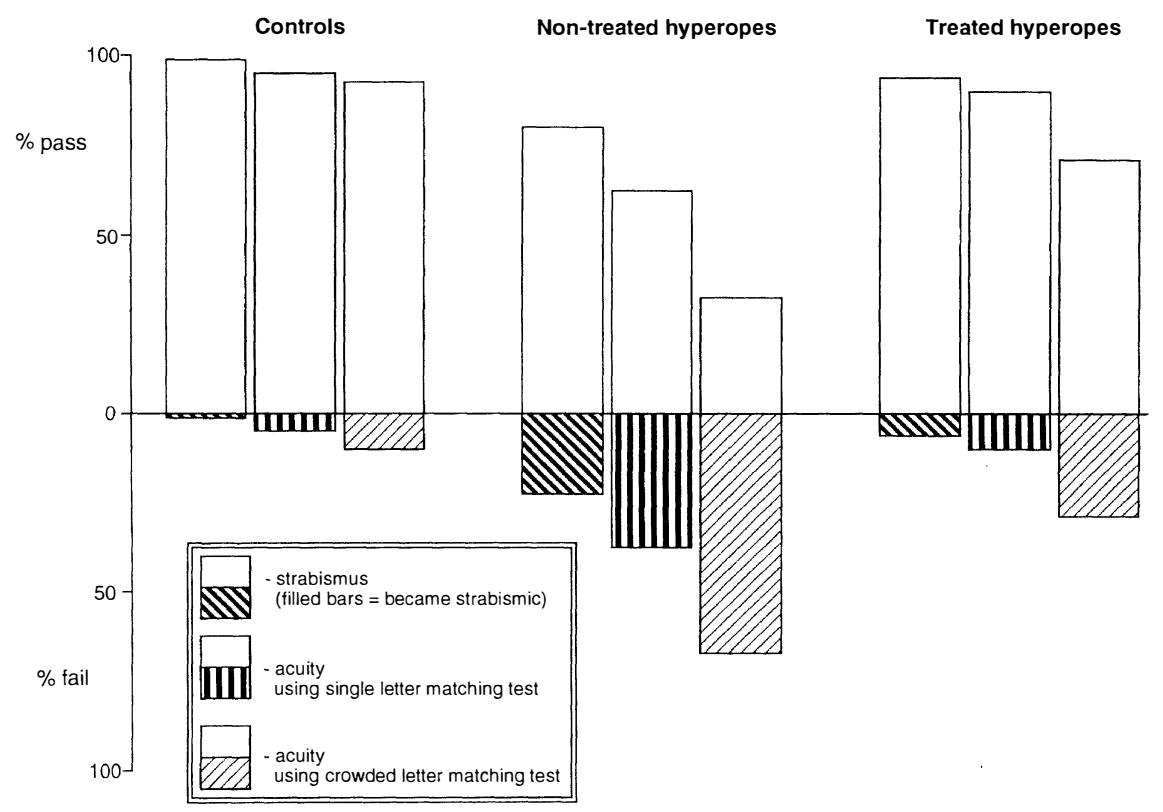

Fig. 3. Outcome measures at 4 years from the first screening programme: strabismus; acuity measured using single letters; acuity measured using the Cambridge Crowding Cards (see text for 'failure' criteria). 


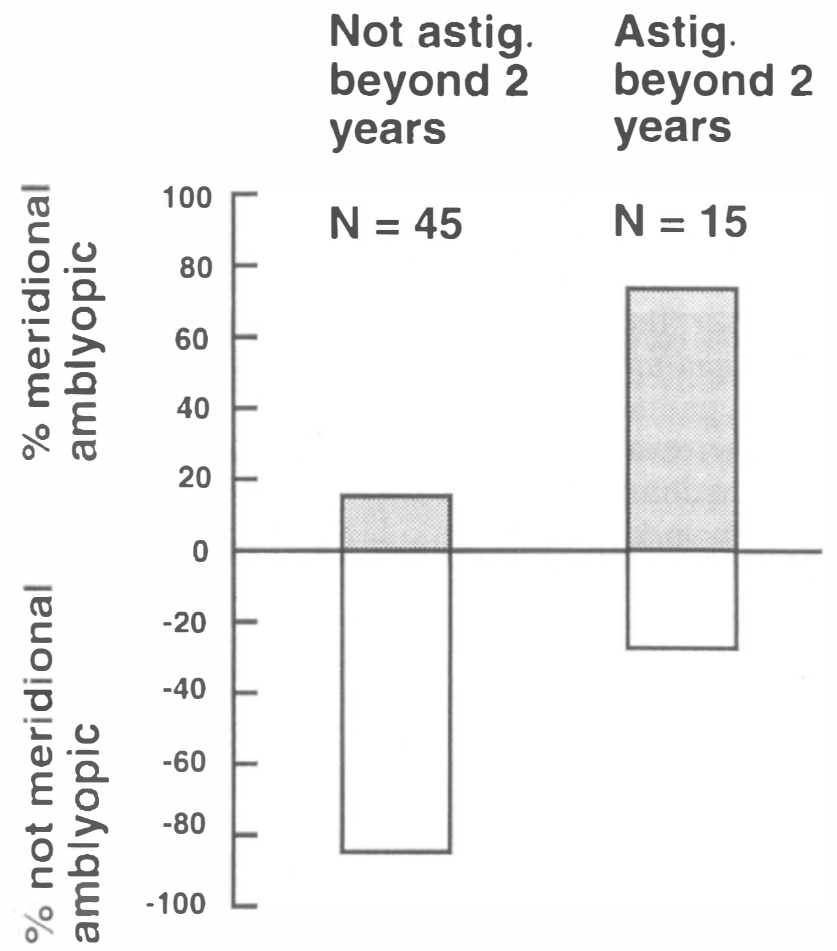

Fig. 4. The incidence of meridional amblyopia at 4 years in infant hyperopes (who have not been corrected with spectacles) in relation to their level of astigmatism at 2 years of age (astigmatism $\geqslant 1.0 D$ at 2 years).

hyperopic group and $11.1 \%(11 / 99)$ of the controls failed to obtain $6 / 9$ equivalent in one or both eyes. Once again there was no significant difference between the controls and treated group but the non-treated group were significantly worse than both the other two groups $(p<0.001)$. Failures on both acuity tests were not necessarily in one eye, as would be expected from strabismic or anisotropic amblyopia. Bilateral acuity deficits, which do not fit with conventional ideas of amblyopia as the result of an interocular competitive process, were common in the untreated hyperopic groups.

An alternative approach to this analysis is to compare the hyperopic groups offered and not offered treatment, regardless of whether the former group actually wore the prescribed correction. The 'not offered' group were found to have a significantly worse outcome than the 'offered' group on all three measures (strabismus, single-letter acuity, crowded acuity). ${ }^{16}$

\section{Meridional Amblyopia}

One possibility is that early astigmatism may be a contributory cause to some cases of amblyopia (since children with large astigmatisms were more likely to appear in the hyperopic group than among the controls). Meridional amblyopia - reduced acuity in one meridian - has been demonstrated in astigmatic adults $^{36}$ and is presumed to be a consequence of

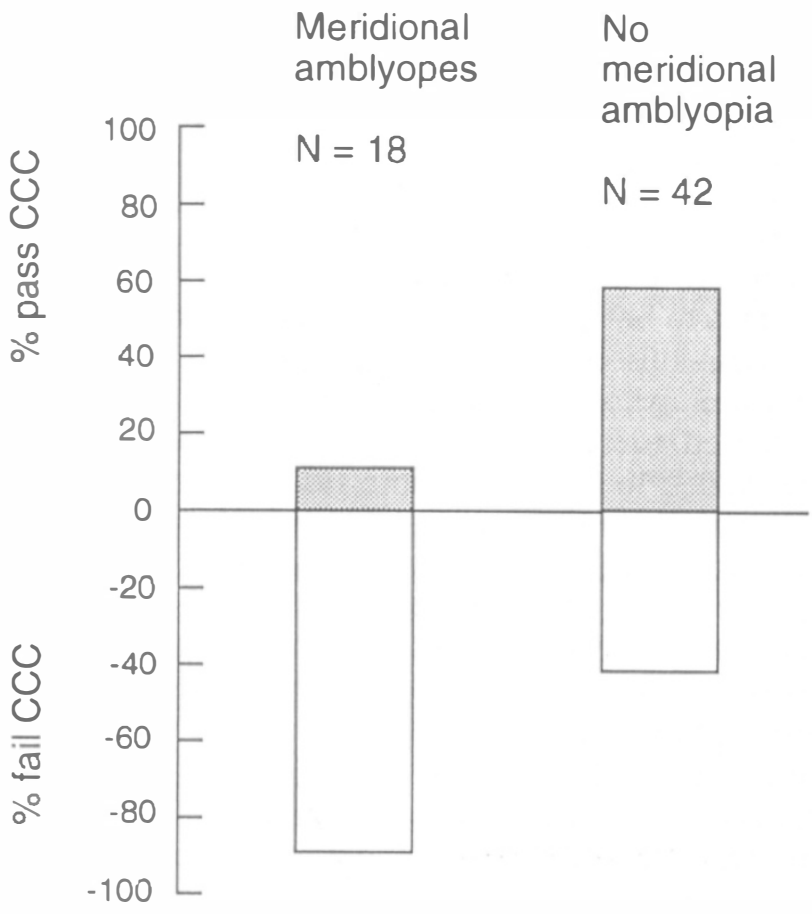

Fig. 5. The relationship between meridional amblyopia and acuity results on the Cambridge Crowding Cards (CCC) at 4 years).

differential image blur caused by marked astigmatism in an early critical period.

Recognition of letter targets could be impaired by poor acuity in any meridian. To examine meridional acuity, we tested a subset of the untreated hyperopic group with grating stimuli in an automated FPL procedure. ${ }^{37}$ Acuity measures were taken for vertical and horizontal gratings in separate short staircase procedures. $^{38}$ The children were tested at 4 years, wearing a full cylinder correction at the time of testing. If a reliable difference of more than 0.67 octaves was found between acuity in the two meridians, the child was deemed 'meridionally amblyopic'. We examined the refractive data over the course of the first 3 years in the same children and divided the group into those who were persistently astigmatic (over $1 \mathrm{D}$ ) after 2 years of age, and those who had lost their astigmatism by 2 years. As can be seen in Fig. 4, there is a strong relationship between those showing meridional amblyopia and persistence of astigmatism. We also compared our measures of meridional amblyopia with the child's performance on the acuity tests at 4 years (Fig. 5). Children who failed the acuity tests tended also to be those who showed meridional amblyopia. However, there is a significant group who failed the letter test but were not meridional amblyopes on our criterion. Our conclusion from these data is that although meridional amblyopia probably contributes to poor 
pre-school acuity in the uncorrected hyperopic group, it is not the only cause.

So why do many of our children who had been infant hyperopes and had not worn correcting spectacles fail the Cambridge Crowding Cards test? It would appear that there is no one single cause. Some are indeed truly amblyopic at 4 years and it remains to be seen if later treatment at this stage can alleviate this condition in this group. Some of the children who had been hyperopic in infancy appeared to fail the crowded acuity task because they failed to understand the task of matching only one letter in the centre of the array. Their behaviour was often rather like that of a younger child in that they would have no difficulty with single-letter matching but when presented with the crowded cards became confused and lost attention. We suspect that some of these children have mild delays that go beyond the visual domain. We are pursuing this possibility in the second programme with followup and measurement of language, motor and visuocognitive ability in these children.

\section{DISCUSSION}

The results of the first programme can be summarised: children who were hyperopic in infancy were 13 times more likely to become strabismic by 4 years than those with refractions closer to emmetropia. They were 6 times more likely to show measurable acuity deficits. If they wore a partial spectacle correction, these risk ratios were reduced to $4: 1$ and 2.5:1 respectively. Thus we support the findings of Ingram ${ }^{2,3}$ that cycloplegic refraction in infancy has a high predictive value for identifying children at risk of strabismus and amblyopia. However, Ingram et al. ${ }^{4}$ did not find that spectacle correction was successful in preventing later amblyopia and strabismus. This difference may be due to differences in the exact criteria and protocol for spectacle prescription, or in the compliance level achieved, and these may be critical factors in deciding the effectiveness of infant screening.

Can children at risk of strabismus and amblyopia be identified without the necessity for cycloplegia at the initial screening? Results of the first follow-up in our second programme (Fig. 1, right) show that accommodative lag, measured using videorefraction without cycloplegia, is strongly predictive of hyperopic refraction under cycloplegia. Comparison of the proportion of the population confirmed as hyperopes in the first and second studies indicates that the noncycloplegic screening procedure is successfully detecting the large majority of the hyperopes. Thus non-cycloplegic screening should be able to predict children at risk of strabismus and amblyopia. However, whether there are differences in outcome between hyperopic infants who show a marked accommodative lag at 8 months, and those who successfully overcome their hyperopia, remains a major question for the final outcome of the second programme.

A likely mechanism contributing to the association between hyperopia in infancy and poor pre-school vision is deprivation amblyopia due to image blur. However, the contributions of blur due to spherical hyperopia and that due to astigmatism have not yet been separated. Strabismus is also predictively linked to hyperopia in infancy. This relationship is often explained in terms of the hypothesis of accommodative estropia, but this hypothesis leaves a good deal more to be explained. The extent to which the developmental visual problems of hyperopes can be alleviated by spectacle correction suggests that the optical effects of hyperopia have a causal role. However, we suspect that hyperopia may be correlated with much more general developmental variables, going beyond purely visual performance; this will be one focus of follow-up measures in the second programme.

Refraction is not constant over the pre-school years, particularly for the hyperopic group. Changes in refraction may be under some control by visual processing, but our study shows no sign of such a process being affected by refractive correction. The relative contributions to emmetropisation of purely maturational processes and of visual feedback will require much more detailed data to disentangle. We can have some confidence, however, that the levels of spectacle correction we have used did not disrupt this developmental process.

Normal, binocular, pattern vision is the result of an interplay of three kinds of factors: optical, in the image formation of the eye; sensori-neural, in the processing of that image and interaction of the images of the two eyes; and muscular, both in the active adjustment of accommodation and in the control of eye movements. The balance of these mechanisms is delicate and the plasticity of the developing system is not always adequate to the task of maintaining it. We hope that the follow-up of the children in the second programme into school age will enable us to understand these interactive processes more fully and to offer treatment and intervention at the appropriate stage of development to maximise the chances of children entering school with normal vision.

A population screening programme is a major undertaking and its benefits, even when proven, have to be evaluated in the broader context of health care needs. It is extremely difficult to quantify the benefits of preventing strabismus and amblyopia. However, a less daunting task is to compare the cost-effectiveness of refractive screening against the surveillance for visual disorders currently offered in primary child 
health care, and an analysis of this kind is planned as a component of the current second programme.

This work has been supported by the Medical Research Council of Great Britain and by a grant from the East Anglia Regional Health Authority. Kim Durden, Jonathan Pointer, Dr David Allen, Jackie Day, Carol Evans, Dr Fiona Griffith, Dr Jocelyn Herrtage, Ann McIntyre, Dr Michael Mair, Dr Kate Petrie, Elizabeth Pimm-Smith, Claire Towler, John Wattam-Bell, Frank Weeks, Francoise Matthieu, Sarah Rae, Fiona Macpherson, Clare Hughes and Jacky Wade have all assisted in carrying out the screening and follow-up. We are grateful to Dr David Vickers and members of the Department of Community Health, Cambridge Health Authority, for their support, and many general practitioners and health visitors for their co-operation. Dr Sue Atkinson (Regional Director of Public Health, South Thames), together with Oliver Braddick and Janette Atkinson, jointly designed the first programme, Dr Sue Atkinson administered a parallel screening programme in Bristol and is involved in costeffectiveness analysis in the second programme.

Key words: Vision screening, Infant vision, Refraction, Hyperopia, Strabismus, Amblyopia.

\section{REFERENCES}

1. Miles FA, Judge SJ. Optically induced changes in the neural coupling between vergence eye movements and accommodation in human subjects. In: Lennerstrand G, Zee DS, Keller E, editors. Functional basis of ocular motility disorders. Oxford: Pergamon Press, 1982.

2. Ingram RM, Traynar MJ, Walker C, Wilson JM. Screening for refractive errors at age 1 year: a pilot study. Br J Ophthalmol 1979;63:243-50.

3. Ingram RM. Refraction as a means of predicting squint and amblyopia in preschool siblings of children known to have these defects. Br J Ophthalmol 1979;63:238-42.

4. Ingram RM, Walker C, Wilson JM, Arnold PE, Lucas J, Dally S. A first attempt to prevent squint and amblyopia by spectacle correction of abnormal refractions from age one year. $\mathrm{Br} \mathrm{J}$ Ophthalmol 1985; 69:851-3.

5. Atkinson J, Braddick OJ, Durden K, Watson PG, Atkinson S. Screening for refractive errors in 6-9 month old infants by photorefraction. Br J Ophthalmol 1984;68:105-12.

6. Castanera de Molina A, Munoz LG, Castanera AS. El metodo de fotorefraccion coaxial isotropica (VPR-1) en la deteccion precoz de la ambliopia. Presented at the X Congreso de la Sociedad Espanola de Estrabologia, Madrid.

7. Somov EE. The videorefractometry for the children of different age on the device of Clement Clarke International Ltd (London). Plenary Meeting of the Leningrad Scientific Medical Society of Ophthalmologists.

8. Atkinson J, Braddick OJ, Ayling L, Pimm-Smith E, Howland HC, Ingram RM. Isotropic photorefraction: a new method for refractive testing of infants. Doc Ophthalmol Proc Ser 1981;30:217-23.

9. Atkinson J, Braddick OJ. The use of isotropic photorefraction for vision screening in infants. Acta Ophthalmol Suppl 1983;157:36-45.

10. Atkinson J, Braddick OJ. Vision screening and photorefraction: the relation of refractive errors to strabismus and amblyopia. Behav Brain Res 1983; 10:71-80.

11. Howland HC, Atkinson J, Braddick OJ, Howland B.
Optics of photorefraction: orthogonal and isotropic methods. J Opt Soc Am 1983;73:1701-8.

12. Kaakinen K. Simultaneous two-flash static photoskiascopy. Acta Ophthalmol (Stockh) 1981;59:378-86.

13. Abramov I, Hainline L, Duckman RH. Screening infant vision with paraxial photorefraction. Optom Vision Sci 1990;67:538-45.

14. Bobier WR, Braddick OJ. Eccentric photorefraction: optical analysis and empirical measures. Am J Optom Physiol Optics 1985;62:614-20.

15. Wesemann W, Norcia AM, Allen D. Theory of eccentric photorefraction (photoretinoscopy): astigmatic eyes. J Opt Soc Am A 1991;8:2038-47.

16. Atkinson J. Infant vision screening: prediction and prevention of strabismus and amblyopia from refractive screening in the Cambridge photorefraction programme. In: Simons K, editor. Early visual development: normal and abnormal. New York: Oxford University Press, 1993.

17. Atkinson J, Braddick OJ, Wattam-Bell J, Durden K, Bobier W, Pointer J, Atkinson S. Photorefractive screening of infants and effects of refractive correction. Invest Ophthalmol Vis Sci (Suppl) 1987;28:399.

18. Atkinson J. Detection and prevention of strabismus and amblyopia in the Cambridge District population infant vision screening population, using isotropic photorefraction and videorefraction. In: Transactions of the European Congress on Paediatric Ophthalmology and Orthoptics, Maastricht, 1994.

19. Anker SE, Atkinson J, Braddick OJ, Ehrlich DL, Weeks F, Wade J. Accommodative measures of ametropia from video-refractive screening of a total infant population. Invest Ophthalmol Vis Sci (Suppl) 1995;36:S48.

20. Atkinson J, Anker SE, Ehrlich DL, Braddick OJ, Rae S, Weeks F, Macpherson F. A non-cycloplegic videorefractive infant population screening programme: design, outcome measures and cost effectiveness. Invest Ophthalmol Vis Sci 1995;36:S868.

21. Atkinson J, Anker SE, Braddick OJ, Ehrlich DL, Wattam-Bell J. Non-cycloplegic videorefraction: applications to infant refractive screening. Strabismus 1994;2(1):42.

22. Braddick OJ, Atkinson J, Wattam-Bell J, Anker S, Norris V. Videorefractive screening of accommodative performance in infants. Invest Ophthalmol Vis Sci (Suppl) 1988;29:60.

23. Howland HC, Atkinson J, Braddick OJ, French J. Infant astigmatism measured by photorefraction. Science 1978;202:331-3.

24. Mohindra I, Held R, Gwiazda J, Brill S. Astigmatism in infants. Science 1978;202:329-31.

25. Atkinson J, Braddick OJ, French J. Infant astigmatism: its disappearance with age. Vision Res 1980;20:891-3.

26. Braddick O. Binocularity in infancy. Eye 1996; 10: 182-8.

27. Banks MS. Infant refraction and accommodation. Int Ophthalmol Clin 1980;20:205-32.

28. Banks MS. The development of visual accommodation during early infancy. Child Dev 1980;51:646-66.

29. Hung L, Crawford MLJ, Smith EL. Spectacle lenses alter eye growth and the refractive status of young monkeys. Nature Med 1995;1:761-3.

30. Ehrlich DL, Atkinson J, Braddick OJ, Bobier W, Durden K. Reduction of infant myopia: a longitudinal cycloplegic study. Vision Res 1995;35:1313-4.

31. Sheridan MD. Manual for the STYCAR vision tests. Slough: NFER Publishing Co., 1976.

32. Hilton AF, Stanley JC. Pitfalls in testing children's 
vision by the Sheridan Gardiner single optotype method. Br J Ophthalmol 1972;56:135-9.

33. Atkinson J, Anker S, Evans C, McIntyre A. The Cambridge Crowding Cards for preschool visual acuity testing. In: Transactions of the 6th International Orthoptic Congress, Harrogate, UK, 1987.

34. Atkinson J, Anker S, Evans C, Hall R, Pimm-Smith E. Visual acuity testing of young children with the Cambridge Crowding Cards at 3 and 6 months. Acta Ophthalmol (Stockh) 1988;66:505-8.

35. Atkinson J, Pimm-Smith E, Evans C, Harding G, Braddick OJ. Visual crowding in young children. Doc Ophthalmol Proc Ser 1986;45:210-3.
36. Mitchell DE, Freeman RD, Millodot M, Haegerstrom G. Meridional amblyopia: evidence for modification of the human visual system by early visual experience. Vision Res 1973;13:535-58.

37. Atkinson J, Braddick OJ, Pimm-Smith E. 'Preferential looking' for monocular and binocular acuity testing of infants. Br J Ophthalmol 1982;66:264-8.

38. Atkinson J, Wattam-Bell J, Pimm-Smith E, Evans C, Braddick OJ. Comparison of rapid procedures in forced choice preferential looking for estimating acuity in infants and young children. Doc Ophthalmol Proc Ser 1986;45:192-200. 